\title{
Phosphorylation of phosphatase and tensin homolog induced by Helicobacter pylori promotes cell invasion by activation of focal adhesion kinase
}

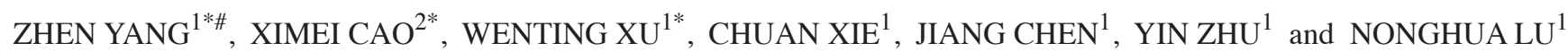 \\ ${ }^{1}$ Department of Gastroenterology, The First Affiliated Hospital of Nanchang University, Nanchang, Jiangxi 330006; \\ ${ }^{2}$ Department of Gastroenterology, First Peoples' Hospital of Jiujiang, Jiujiang, Jiangxi 332000, P.R. China
}

Received January 15, 2016; Accepted October 18, 2017

DOI: $10.3892 / \mathrm{ol} .2017 .7430$

\begin{abstract}
Phosphorylation of the phosphatase and tensin homolog (PTEN) tumor suppressor at Ser380/Thr382/Thr383 residues is a novel mechanism underlying PTEN inactivation in gastric carcinogenesis, which may be triggered by Helicobacter pylori infection. To investigate this further, the effect of $H$. pylori infection on PTEN phosphorylation and the subsequent activation of focal adhesion kinase (FAK), were evaluated in gastric tissue samples from Mongolian gerbils and in the human gastric epithelial mucosa cell line GES-1 using immunohistochemistry, western blotting and Transwell assays. The in vivo and in vitro results of the present study demonstrated that $H$. pylori infection induced the phosphorylation and inactivation of PTEN at Ser380/Thr382/383, and the subsequent phosphorylation and activation of FAK at Tyr397. Gastric epithelial cell invasion was also increased. Furthermore, stable expression of a dominant-negative PTEN mutant inhibited the enhanced FAK activation and cell invasion induced by $H$. pylori infection. These results suggest that the mechanism underlying $H$. pylori-induced carcinogenesis may involve promoting cell invasion through the phosphorylation of PTEN and the activation of FAK.
\end{abstract}

Correspondence to: Dr Nonghua Lu, Department of Gastroenterology, The First Affiliated Hospital of Nanchang University, 17 Yongwaizheng Street, Nanchang, Jiangxi 330006, P.R. China

E-mail: lunonghua@ncu.edu.cn

*Contributed equally

"Deceased

Abbreviations: PTEN, phosphatase and tensin homolog; FAK, focal adhesion kinase; FBS, fetal bovine serum; DMEM, Dulbecco's modified Eagle's medium

Key words: Helicobacter pylori, phosphatase and tensin homolog, focal adhesion kinase, phosphorylation, cell invasion

\section{Introduction}

Helicobacter pylori is a gram-negative bacterial species that represents the most common bacterial infection of the gastric epithelium worldwide $(1,2)$. H. pylori colonization is the most prominent identified risk factor for malignancies that arise within the stomach, and it increases the risk of atrophic gastritis, intestinal metaplasia, and distal gastric adenocarcinoma (3-6). H. pylori infection has been classified by the World Health Organization as a carcinogen for the development of gastric carcinoma (7); this cancer type is the second most common cause of cancer-associated mortality worldwide, accounting for $\sim 989,600$ new cases and $\sim 738,000$ mortalities per year, half of which occur in eastern Asia $(8,9)$.

Since the initial description of $H$. pylori by Marshall and Warren in 1984 (10), significant advances towards understanding gastric carcinogenesis have been achieved. Gastric carcinogenesis is a multifactorial and multistep process that involves the activation of oncogenes and the inactivation of tumor suppressor genes (11-16). One such tumor suppressor gene is phosphatase and tensin homolog (PTEN) (17-19), which has lipid and protein phosphatase activities. PTEN inactivation has been observed in glioblastomas, endometrial carcinomas and skin, prostate and breast cancers (17-19). Previous studies have indicated that PTEN inactivation in gastric cancers may be due to genetic or epigenetic changes, including mutation, loss of heterozygosity, promoter hypermethylation, regulation of microRNA and post-translational phosphorylation (14,20-25). Phosphorylation of PTEN on sites within the C2 domain (Ser380, Thr382 and Thr383) decreases phosphatase activity and increases stability $(26,27)$, which may cause loss of tumor suppressor function and increased susceptibility to cancer. In our previous study, it was demonstrated that PTEN phosphorylation at residues Ser380/Thr382/ Thr383 is a novel mechanism underlying PTEN inactivation in gastric carcinogenesis $(14,28)$. This was observed to be triggered by $H$. pylori infection and required for the activation of the phosphatidylinositol-4,5-bisphosphate 3-kinase/protein kinase B signaling pathway and the subsequent promotion of cell survival (14).

PTEN regulates a variety of other downstream signaling pathways, including the regulation of cell migration, invasion 
and growth by focal adhesion kinase (FAK) (29-32). FAK is a key molecule that is implicated in integrin signaling, which contributes to cancer progression, invasion and metastasis $(29,33,34)$. Previous studies have identified that H. pylori may induce FAK activation and cytoskeletal reorganization $(35,36)$. Therefore, the aim of the present study was to determine the effect of $H$. pylori-induced PTEN phosphorylation on the activation of FAK in vivo and in vitro, in order to identify the mechanisms underlying gastric cancer development.

\section{Materials and methods}

H. pylori strain. As previously described $(14,28)$, the $\mathrm{CagA}^{+}$ and $\mathrm{Vag}^{+} H$. pylori strain ATCC43504 (National Institute for Communicable Diseases and Prevention of Chinese Center for Disease Control and Prevention, Beijing, China) was cultured on Campylobacter agar plates (Shanghai Municipal Center For Disease Control and Prevention, Shanghai, China) supplemented with $10 \%$ sheep blood (Shanghai Kangfeng, Biological Technology Co., Ltd, Shanghai, China) and incubated at $37^{\circ} \mathrm{C}$ under microaerophilic conditions $\left(5 \% \mathrm{O}_{2}, 10 \% \mathrm{CO}_{2}\right.$ and $85 \% \mathrm{~N}_{2}$ ) for $24 \mathrm{~h}$, then subcultured in Brucella broth (Shanghai Municipal Center For Disease Control and Prevention) supplemented with $10 \%$ fetal bovine serum (FBS; Gibco; Thermo Fisher Scientific Inc., Waltham, MA, USA) at $37^{\circ} \mathrm{C}$ under a microaerophilic atmosphere for $16-18 \mathrm{~h}$. Bacterial density was estimated spectrophotometrically as the absorbance at $600 \mathrm{~nm}$ [optical density $(\mathrm{OD})_{600}$, and viable counts were determined as colony-forming units $(\mathrm{CFU}) / \mathrm{ml}\left(1 \mathrm{OD}_{600}=10^{9} \mathrm{CFU} / \mathrm{ml}\right)$.

Mongolian gerbils. A total of 79 specific pathogen-free male Mongolian gerbils (age, 5-8 weeks; weight, 30-50 g) purchased from the Zhejiang Academy of Medical Sciences (Hangzhou, China), were maintained in an isolated clean room with regulated temperature $\left(20-22^{\circ} \mathrm{C}\right)$, humidity $(\sim 55 \%)$ and a 12/12-h light/dark cycle with ad libitum rodent diet and water. As previously described $(14,28)$, following one week of observation, the gerbils were administered $1 \mathrm{ml}$ orogastric infusions of sterile Brucella broth $(\mathrm{n}=25$; controls) or $1 \times 10^{9} \mathrm{CFU}$ of $H$. pylori $(\mathrm{n}=54)$ once every three days, for a total of 10 infusions. Gerbils were fasted for $12 \mathrm{~h}$ prior to $H$. pylori inoculation and drinking water was withheld following the inoculation. Food and water were freely available to the gerbils at $4 \mathrm{~h}$ post-inoculation. The animals were euthanized using sodium pentobarbital $(150 \mathrm{mg} / \mathrm{kg})$ and cervical dislocation at 6 months $(H$. pylor $i$-infected, $\mathrm{n}=30$; controls, $\mathrm{n}=15)$ or 12 months (H. pylori-infected, $\mathrm{n}=24$; controls, $\mathrm{n}=10$ ). Then gastric tissue was separated from the animals, which were cut along the greater curvature of stomach, the gastric tissue was smoothed out and linear strips of gastric tissue extending from the squamocolumnar junction through the proximal duodenum were collected. Detection of $H$. pylori infection was carried out using Giemsa staining (14). All animal experiments and procedures were approved by the Ethics Committee of The First Affiliated Hospital of Nanchang University (Nanchang, China).

Cell line and lentivirus infection. The GES-1 immortalized human gastric epithelial mucosa cell line from the Beijing
Institute for Cancer Research (Beijing, China) was cultured in Dulbecco's modified Eagle's medium (DMEM; Hyclone, Logan, Utah, USA) supplemented with $10 \%$ FBS, $100 \mathrm{U}$ penicillin and $100 \mu \mathrm{g} / \mathrm{ml}$ streptomycin (Gibco; Thermo Fisher Scientific Inc., Waltham, MA, USA) at $37^{\circ} \mathrm{C}$ in an atmosphere containing $5 \% \mathrm{CO}_{2}$. DMEM supplemented with $10 \%$ FBS to suspend the $H$. pylori was added to infect GES- 1 cells with various multiplicity of infection (MOI) for $0,0.5,1,3$ and $6 \mathrm{~h}$. The same volume of DMEM $(5 \mathrm{ml})$ supplemented with $10 \%$ FBS was added to GES-1 cells in the control group.

Wild-type PTEN, dominant-negative mutant PTEN (C124S) and empty lentiviral supernatants were purchased from Invitrogen (Thermo Fisher Scientific, Inc.). For lentiviral infection, GES-1 cells were cultured to $\sim 60 \%$ confluence and incubated with the aforementioned three lentiviral supernatants, respectively, and hexadimethrine bromide (Sigma-Aldrich, St. Louis, MO, USA) for $6 \mathrm{~h}$ as aforementioned. After $48 \mathrm{~h}$, the cells were split and cultured in selection media supplemented with blasticidin ( $6 \mu \mathrm{g} / \mathrm{ml}$; Sigma-Aldrich) for a further 2 weeks at $37^{\circ} \mathrm{C}$ in an atmosphere containing $5 \% \mathrm{CO}_{2}$. The stable cell lines expressing a wild type or dominant-negative mutant PTEN were then established. As previously described, PTEN was overexpressed in stable cell lines carried with wild-type PTEN (28). Dominant-negative mutant PTEN (C124S) contributed to the phosphorylation and inactivation of PTEN at residues Ser380/Thr382/383. In addition, the empty vector did not contain exogenous gene.

Immunohistochemistry. Immunohistochemistry was performed on paraffin-embedded sections of Mongolian gerbil gastric tissues using anti-PTEN (cat. no. ab31392; dilution 1:150), anti-phosphorylated (p)-PTEN (Ser380/Thr382/Thr383; cat. no. ab47332; 1:800), anti-FAK (cat. no. ab40794; 1:400), and anti-p-FAK (Tyr397; cat. no. ab4803; 1:400) antibodies (all from Abcam, Cambridge, UK) and polyperoxidase-conjugated anti-mouse or rabbit immunoglobulin $\mathrm{G}$ antibody as provided in PV-9000 Polymer Detection System (Zhongshan Goldenbridge, Beijing, China) using previously described methods $(12,14,15)$. The stained sections were selected, reviewed and scored from five randomly selected high-power fields (40x objective lens) by two pathologists blinded to the histopathological data. Grading discrepancies were re-reviewed and discussed to obtain a final score. Epithelial cells with yellow or brown staining in the nucleus and/or cytoplasm were defined as positive for immunoreactivity. The percentage of immunoreactive cells from 100 cells selected for each field, were averaged from the five fields and scored as follows: $0, \leq 5.0 \%$; $1,5.1-25.0 \% ; 2,25.1-50.0 \% ; 3,50.1-75.0 \%$; and $4,>75.1 \%$ immunoreactivity. Furthermore, the staining intensity was also semi-quantitatively assessed as follows: 0 , no staining; 1 , weak staining; 2 , moderate staining; and 3 , intense staining. The overall protein expression level was presented as a grade calculated from the integrated scores of the 'area $x$ intensity', as follows: Grade 1, score 0-2 (negative); grade 2, score 3-5 (weakly positive); grade 3 , score 6-8 (moderately positive); and grade 4, score 9-12 (intensely positive).

Immunoblotting. Western blotting was performed according to standard methods as described previously (13) using 
A 6 Months 12 Months
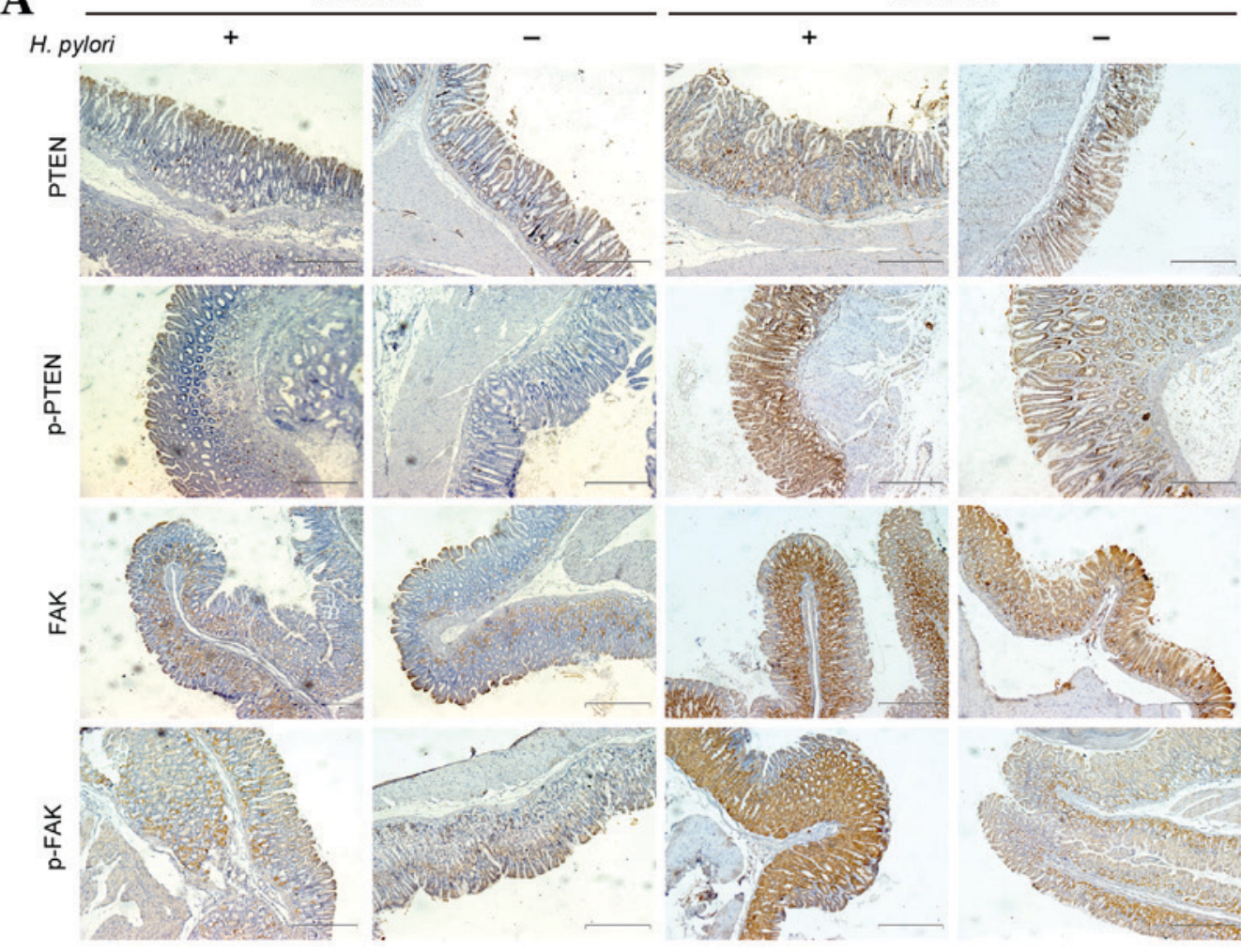

B

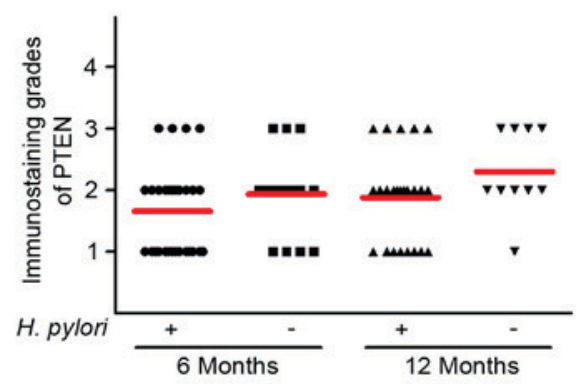

D

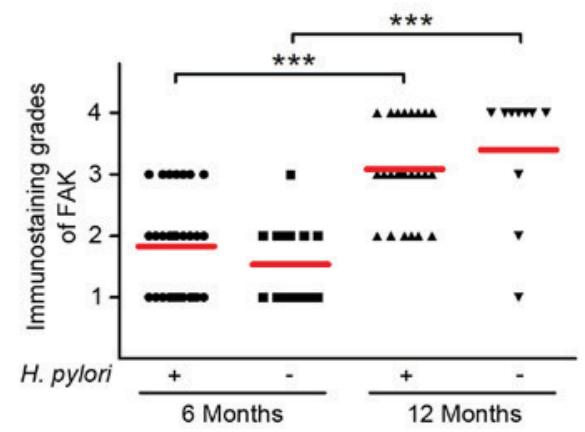

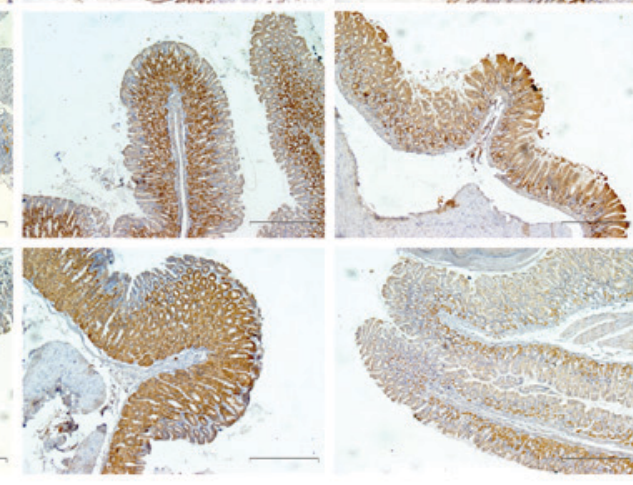

C

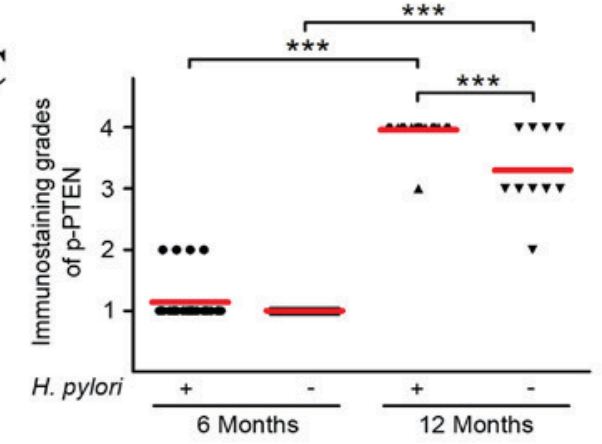

E

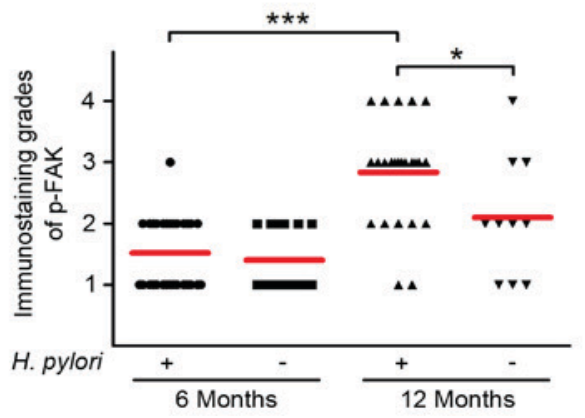

Figure 1. Immunohistochemical staining of PTEN, p-PTEN, FAK and p-FAK in the gastric mucosal regions of Mongolian gerbils. Gastric tissue sections from $H$. pylori-infected gerbils were collected at 6 or 12 months following inoculation and stained using antibodies against PTEN, p-PTEN, FAK and p-FAK. (A) Representative images of immunohistochemical staining are shown (scale bars, $200 \mu$ m). Immunoreactivity levels of (B) PTEN, (C) p-PTEN, (D) FAK and (E) p-FAK in the tissues were semi-quantitatively assessed, and the protein expression levels were expressed as grades 1-4. Mean grades (red lines) for each protein in each group are shown. ${ }^{*} \mathrm{P}<0.05 ;{ }^{* * *} \mathrm{P}<0.001$. PTEN, phosphatase and tensin homolog; p-, phosphorylated; FAK, focal adhesion kinase; H. pylori, Helicobacter pylori.

anti-PTEN (cat. no. 9559; Cell Signaling Technology, Inc., Danvers, MA, USA; dilution 1:10,000), anti-p-PTEN (Ser380/Thr382/Thr383; cat. no. 9554; Cell Signaling Technology, Inc.; dilution 1:10,000), anti-FAK (cat. no. ab40794; Abcam; 1:1,000), anti-p-FAK (Tyr397; cat. no. ab4803; Abcam; $1: 1,000)$ and anti- $\beta$-actin (sc-1615-R; 1:1,000; Santa Cruz Biotechnology, Inc., Dallas, TX, USA) antibodies. Quantity One software (Bio-Rad Laboratories, Inc., Hercules, CA, USA; version 4.5.1) was used for densitometric measurements of band intensities. 
A
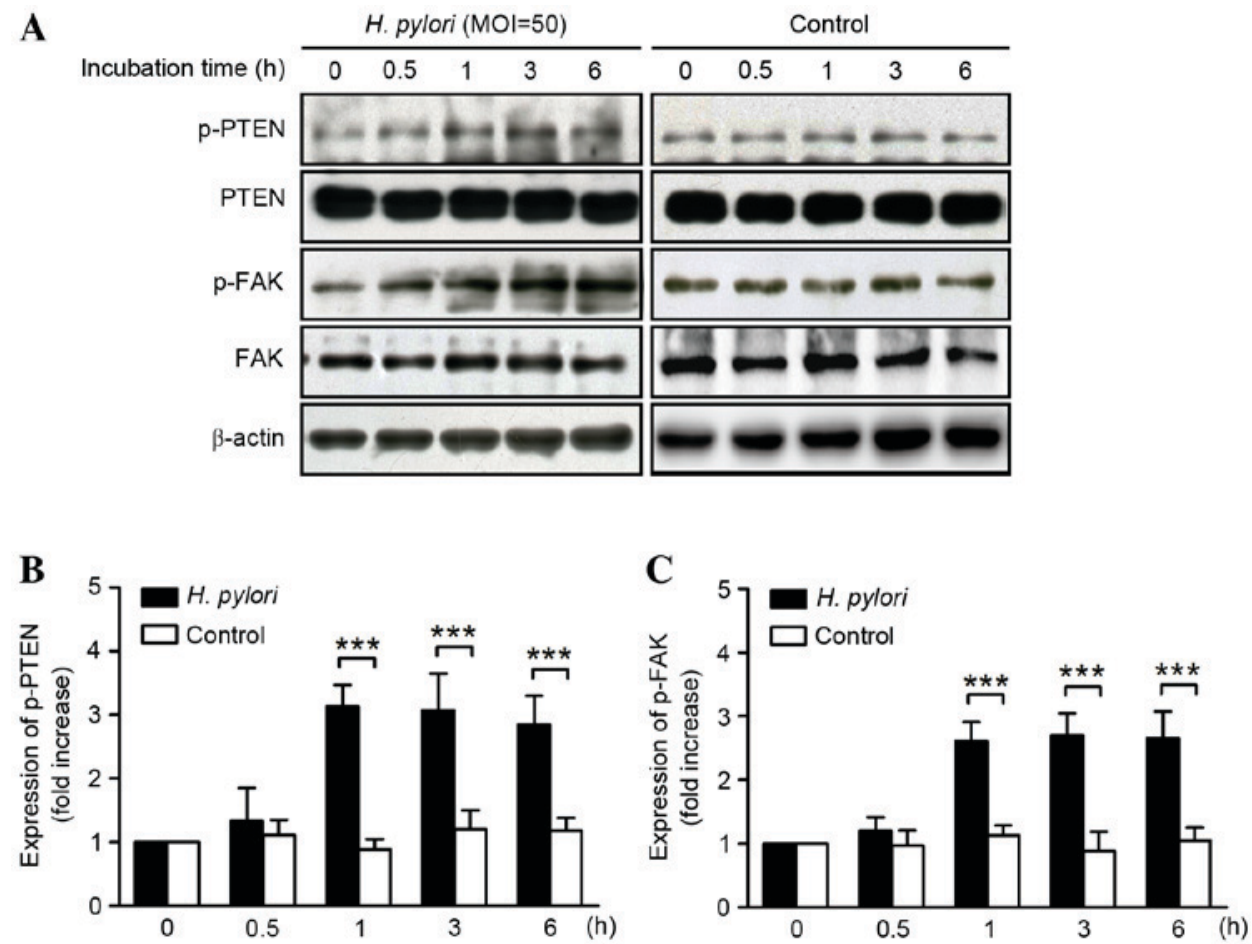

Figure 2. Expression levels and phosphorylation of PTEN and FAK in GES-1 gastric epithelial cells following incubation with H.pylori. (A) Representative western blots from GES-1 cell lysates following incubation with $H$. pylori for $0,0.5,1,3$ and $6 \mathrm{~h}$ (MOI, 50) are shown. Graphs represent quantitative analysis (expressed as the fold of the control) of (B) p-PTEN and (C) p-FAK expression levels; data are presented as the mean \pm standard deviation of triplicated experiments. ${ }^{* * * *} \mathrm{P}<0.001$. PTEN, phosphatase and tensin homolog; p-, phosphorylated; FAK, focal adhesion kinase; H. pylori, Helicobacter pylori.

Invasion assays. GES-1 cells were seeded at a density of $1 \times 10^{4}$ cells/well in Transwell chambers $(8.0 \mu \mathrm{m}$ pore size; Corning Inc., Corning, NY, USA) coated with Matrigel (BD Biosciences, Franklin Lakes, NJ, USA), with medium supplemented with $10 \%$ FBS in the lower chamber as the chemoattractant and medium supplemented with $0.2 \%$ BSA in the upper chamber, then incubated for $20 \mathrm{~h}$ at $37^{\circ} \mathrm{C}$ with $5 \%$ $\mathrm{CO}_{2}$. Cells that did not migrate through the pores were manually removed with a cotton swab. Cells adhered to the bottom of the chamber membrane were fixed in methanol at room temperature for $5 \mathrm{~min}$ and stained with hematoxylin. In three independent experiments, the number of migrated cells on the lower surface of the membrane was counted in 10 visual fields per well using a light microscope at $\mathrm{x} 400$ magnification and means of these counts were analyzed for statistical significance.

Statistical analysis. The data are presented as the mean \pm standard deviation or the fold of the control. Statistical analysis was performed using SPSS 17.0 software (SPSS, Inc., Chicago, IL, USA). Student's t-tests or Mann-Whitney U tests were used to compare the differences in the mean value between various groups. $\mathrm{P}<0.05$ was considered to indicate a statistically significant difference.

\section{Results}

H. pylori infection increases PTEN and FAK phosphorylation in vivo. The Mongolian gerbils were successfully infected with $H$. pylori, which was confirmed by Giemsa staining. No animals challenged with Brucella broth alone exhibited detectable evidence of $H$. pylori infection.
Immunohistochemical analysis revealed no significant difference in PTEN expression levels from $H$.pylori infection at 6 or 12 months following inoculation (Fig. 1). Inhibitory p-PTEN demonstrated variable protein expression levels in all groups, with the exception of the control group after 6 months. The expression levels of p-PTEN were significantly higher in the gastric mucosae of Mongolian gerbils at 12 months following H. pylori infection $(\mathrm{P}=0.000)$ compared with the control, but not in the corresponding groups after 6 months. The PTEN expression levels were higher at 12 months than at 6 months with or without $H$. pylori infection. Immunohistochemical analysis of FAK revealed no difference in expression levels from $H$. pylori infection after 6 or 12 months compared with the controls; however, the expression levels were significantly higher at 12 months compared with at 6 months in the groups with or without $H$. pylori infection ( $\mathrm{P}=0.000$; Fig. 1). By contrast, after 6 months, active p-FAK expression levels were not observed to be altered by $H$. pylori infection compared with the control group, whereas the expression levels were significantly higher compared with the controls after 12 months $(\mathrm{P}=0.041)$. Furthermore, statistical analysis demonstrated that p-FAK expression levels in $H$. pylori-infected gerbils were significantly higher after 12 months compared with 6 months infection $(\mathrm{P}=0.000)$.

H. pylori increases PTEN and FAK phosphorylation in vitro. Western blot analysis of protein lysates from GES-1 cells demonstrated that incubation with $\mathrm{H}$. pylori increased the phosphorylation of PTEN from $1 \mathrm{~h}$, which was observed to be maintained for $\leq 6 \mathrm{~h}(\mathrm{P}<0.001)$ without altering the total PTEN protein expression levels (Fig. 2A). Relative to $0 \mathrm{~h}$, 

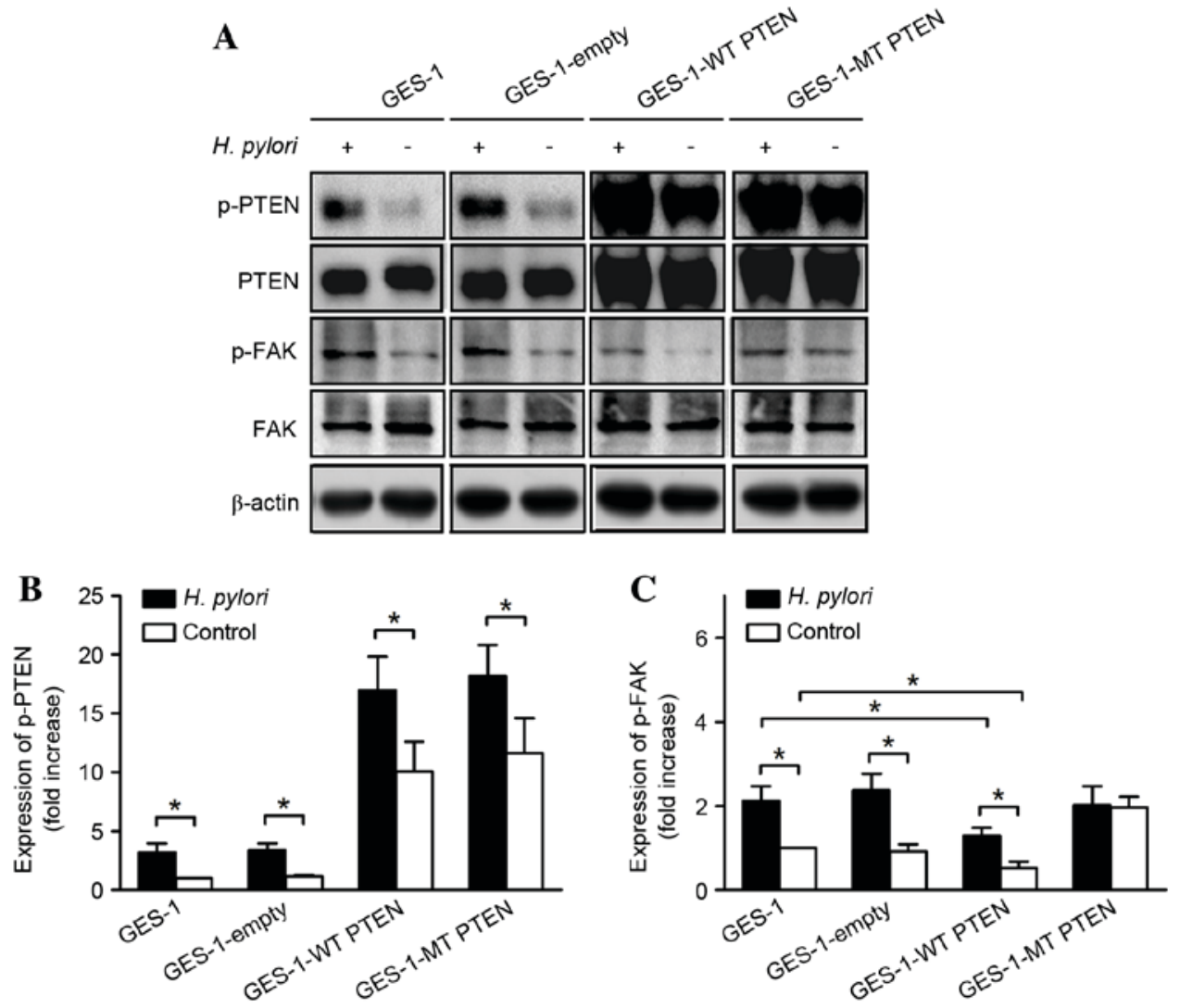

Figure 3. Protein and phosphorylation expression levels of PTEN and FAK following incubation with $H$.pylori in GES-1 gastric epithelial cells overexpressing $\mathrm{mt}$ and wt PTEN constructs. (A) Representative western blots from cell lysates of GES-1 cells expressing wt and mt PTEN constructs (GES-1-WT PTEN and GES-1-MT PTEN, respectively) with and without a 1-h incubation with H. pylori (multiplicity of infection, 50) are shown. Graphs represent quantitative analysis of (B) p-PTEN and (C) p-FAK expression levels; data are presented as the mean \pm standard deviation of triplicated experiments (expressed as the fold of the control). "P<0.05. PTEN, phosphatase and tensin homolog; p-, phosphorylated; FAK, focal adhesion kinase; H. pylori, Helicobacter pylori; mt, mutant; wt, wild type.

the expression levels of p-PTEN were $1.33 \pm 0.62,3.13 \pm 0.34$, $3.07 \pm 0.58$, and $2.85 \pm 0.45$ at $0.5,1,3$ and $6 \mathrm{~h}$, respectively (Fig. 2B). Similarly, the expression levels of p-FAK were $1.22 \pm 0.22,2.62 \pm 0.30,2.70 \pm 0.35$ and $2.65 \pm 0.62$ relative to $0 \mathrm{~h}$ after $0.5,1,3$ and 6 h, respectively (Fig. 2C). The increase was determined to be significant after 1-6 h of GES-1 cell incubation with $H$. pylori $(\mathrm{P}<0.001)$ without affecting the total FAK protein expression.

Role of PTEN in H. pylori-induced phosphorylation of $F A K$. Analysis of the western blots revealed that the relative p-PTEN/PTEN expression levels in GES-1 cells transfected with an empty vector were equal to those in the untransfected control GES-1 cells, while the relative expression levels were significantly increased by $H$. pylori infection in all groups $(\mathrm{P}=0.025)$ (Fig. 3). Overexpression of wild type PTEN markedly inhibited FAK phosphorylation in the GES-1-wild type PTEN group compared with that in the empty vector and untransfected group; although $H$. pylori increased the levels of p-FAK, this increase was significantly suppressed by overexpression of wild type PTEN, compared with the untransfected group $(\mathrm{P}=0.023)$. However, overexpression of an inactive mutant PTEN construct (which mimics phosphorylation at Ser380/Thr382/Thr383) blocked the H.pylori-induced increase in p-FAK, without affecting total FAK expression levels. In addition, this overexpression of mutant PTEN increased basal

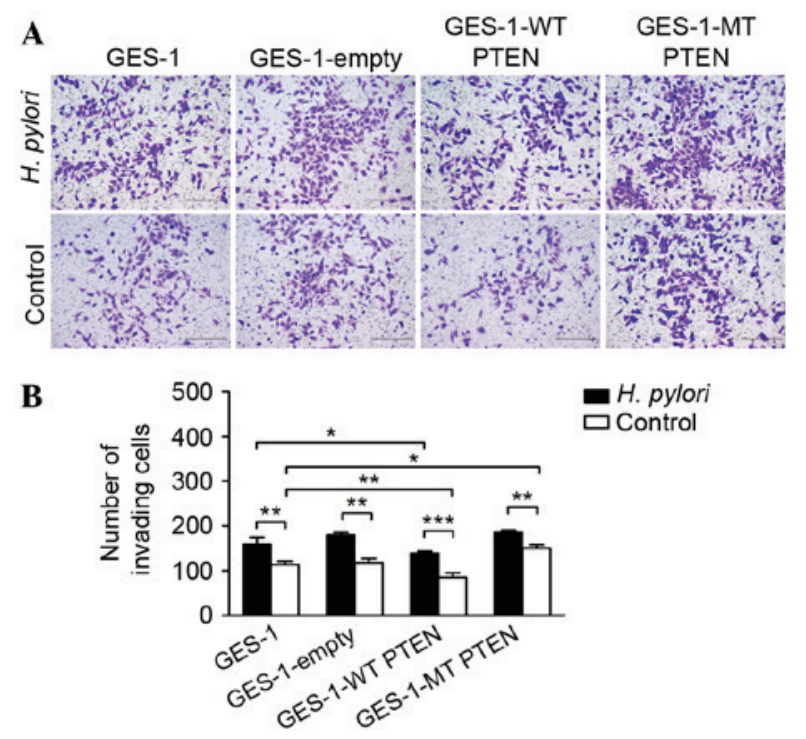

Figure 4. Invasion assay of GES-1 gastric epithelial cells overexpressing mt or wt PTEN constructs following incubation with $H$. pylori. (A) Representative micrographs showing the Transwell invasion of hematoxylin-stained GES-1 cells expressing wt or mt PTEN constructs (GES-1-WT PTEN and GES-1-MT PTEN, respectively), with and without incubation with $H$. pylori (multiplicity of infection, 50; scale bar, $200 \mu \mathrm{m}$ ). (B) Numbers of cells invaded through the membranes are presented as the mean \pm standard deviation of triplicated experiments. ${ }^{*} \mathrm{P}<0.05 ;{ }^{* *} \mathrm{P}<0.01 ;{ }^{* * *} \mathrm{P}<0.001$. PTEN, phosphatase and tensin homolog; p-, phosphorylated; FAK, focal adhesion kinase; H. pylori, Helicobacter pylori; mt, mutant; wt, wild type. 
expression levels of p-FAK, thereby mimicking the effects of H. pylori infection.

Role of PTEN in H. pylori-induced cell invasion. Incubation of GES-1 cells with $H$. pylori increased the number of cells that migrated through a Transwell membrane $(159.03 \pm 15.96$ vs. 113.67 \pm 7.23 ; $P=0.002$; Fig. 4). Similarly, invasion was enhanced by $H$. pylori infection in GES- 1 cells transfected with an empty control vector $(179.67 \pm 6.02$ vs. $118.10 \pm 9.00$; $\mathrm{P}=0.001)$, wild type PTEN $(138.97 \pm 5.00$ vs. $85.06 \pm 10.00$; $\mathrm{P}=0.001)$ and mutant $\mathrm{PTEN}(185.87 \pm 4.93$ vs. $150.32 \pm 7.57$; $\mathrm{P}=0.002)$. Following $H$. pylori infection, the number of invading cells overexpressing wild type PTEN was decreased compared with the empty vector group $(\mathrm{P}=0.001)$. In addition, there was a significant difference among the GES-1-wild type PTEN, mutant PTEN and the untransfected group, as wild type PTEN overexpression decreased the number of invading cells, whereas mutant PTEN expression levels increased the number of invading cells compared with the untransfected group $(\mathrm{P}=0.004)$.

\section{Discussion}

PTEN is recognized as a frequently inactivated tumor suppressor in a variety of human cancers, and several lines of evidence suggest that abnormal activation of FAK is associated with tumor development (37-39). Continuing from our previous study (28), which described the H.pylori-induced phosphorylation of PTEN, the results of the present study demonstrate that gastric cell invasion is promoted by this process via the activation of FAK.

H.pylori infection is one of the most significant identified risk factors for gastric carcinogenesis. Numerous studies use animal models to investigate $H$. pylori infection-associated diseases. The use of various animal species and bacterial strains results in a large variability in the reported results (40). Mongolian gerbils are a unique model due to their low incidence of naturally occurring gastritis and bacterial disease, and have, therefore, commonly been used for studying infection with a standard strain of H.pylori (ATCC43504) (41). The results of the current study demonstrate that, concordant with previous results from patients (28), 6-12 months of infection in these gerbils did not significantly alter PTEN expression levels. However, H. pylori infection did enhance the expression levels of p-PTEN and p-FAK in gerbils. Although FAK expression levels increased with age, they were not affected by $H$. pylori infection.

The in vitro experiments confirmed that $H$.pylori-induced PTEN inactivation promotes FAK phosphorylation, which is consistent with the results of previous studies $(34,35)$. By overexpressing an inactive form of PTEN, where phosphatase activity is eliminated by the mutation of a cysteine to a serine at position 124 (42), the present study demonstrated that the $\mathrm{H}$. pylori-induced phosphorylation of FAK is dependent on PTEN activity. In addition, the overexpression of wild type PTEN reduced basal p-FAK levels, indicating enhanced phosphatase activity. These results are concordant with those of a previous study (39) which indicated that overexpression of PTEN inhibits the invasion and metastasis of gastric cancer. However, FAK expression in that study was reported to be downregulated, which was not observed in the current study.
PTEN is known to be crucially involved in the regulation of cell proliferation, growth and survival, and in tumorigenesis (24); however, the role of PTEN in cancer invasion and metastasis is not well understood. The results of the current study demonstrate that $H$. pylori infection significantly increases gastric epithelial cell invasion. Furthermore, cell invasiveness was found to be dependent on PTEN activity, as it was suppressed by wild type, and enhanced by mutant, PTEN overexpression. However, H. pylori infection was still able to increase the invasion of cells overexpressing mutant PTEN, suggesting the involvement of an additional signaling pathway, including one that may act directly on the cell cytoskeleton, matrix metalloproteinase-7 or integrins (43-45).

Previous reports have suggested that gastric carcinogenesis is associated with dysregulation of the PTEN signaling pathway $(13,14,24,25)$. The results of the present study demonstrate that $H$. pylori infection enhances this dysregulation by promoting the phosphorylation of PTEN, which subsequently increases FAK activation and gastric epithelial cell invasion. Therefore, we propose that early $H$. pylori infection may influence gastric cancer development by altering signaling pathways important for the morphological changes, adhesion, migration and proliferation of gastric mucosal cells.

\section{Acknowledgements}

This study was supported by grants from the National Natural Science Foundation of China (grant nos. 81060038 and 81460377), the Natural Science Foundation of Jiangxi Province, China (grant nos. 20142BAB215036 and 20151BAB205041), the Science and Technology Foundation of Department of Education of Jiangxi Province, China (grant no, GJJ14169), the National Science and Technology Major Projects program for 'Major New Drugs Innovation and Development' of China (grant no. 2011ZX09302-007-03) and the 'Talent 555 Project' of Jiangxi Province, China. The authors would like to thank Professor Ke Yang at the Beijing Institute for Cancer Research for providing the GES-1 immortalized gastric epithelial mucosa cell line, and Professor Zhang Jianzhong of the National Institute for Communicable Diseases and Prevention of Chinese Center for Disease Control and Prevention for providing the H. pylori type strain ATCC43504.

\section{References}

1. Moss SF and Sood S: Helicobacter pylori. Curr Opin Infect Dis 16: 445-451, 2003.

2. Peek RM Jr and Crabtree JE: Helicobacter infection and gastric neoplasia. J Pathol 208: 233-248, 2006.

3. Uemura N, Okamoto S, Yamamoto S, Matsumura N, Yamaguchi S, Yamakido M, Taniyama K, Sasaki N and Schlemper RJ: Helicobacter pylori infection and the development of gastric cancer. N Engl J Med 345: 784-789, 2001.

4. Nomura A, Stemmermann GN, Chyou PH, Kato I, Perez-Perez GI and Blaser MJ: Helicobacter pylori infection and gastric carcinoma among Japanese Americans in Hawaii. N Engl J Med 325: 1132-1136, 1991.

5. Parsonnet J, Friedman GD, Vandersteen DP, Chang Y, Vogelman JH, Orentreich N and Sibley RK: Helicobacter pylori infection and the risk of gastric carcinoma. N Engl J Med 325: 1127-1131, 1991.

6. Correa P: Helicobacter pylori and gastric cancer: State of the art. Cancer Epidemiol Biomarkers Prev 5: 477-481, 1996. 
7. No authors listed: Schistosomes, liver flukes and Helicobacter pylori. IARC Working Group on the Evaluation of Carcinogenic Risks to Humans. Lyon, 7-14 June 1994. IARC Monogr Eval Carcinog Risks Hum 61: 1-241, 1994.

8. Ferlay J, Shin HR, Bray F, Forman D, Mathers C and Parkin DM: Estimates of worldwide burden of cancer in 2008: GLOBOCAN 2008. Int J Cancer 127: 2893-2917, 2010.

9. Jemal A, Bray F, Center MM, Ferlay J, Ward E and Forman D: Global cancer statistics. CA Cancer J Clin 61: 69-90, 2011.

10. Marshall BJ and Warren JR: Unidentified curved bacilli in the stomach of patients with gastritis and peptic ulceration. Lancet 1: $1311-1315,1984$

11. Zhu Y, Shu X, Chen J, Xie Y, Xu P, Huang DQ and Lu NH: Effect of Helicobacter pylori eradication on oncogenes and cell proliferation. Eur J Clin Invest 38: 628-633, 2008

12. Yang Z, Shu X, Chen L, Chen J, Xie Y and Lu NH: Expression of p53-MDM2 feedback loop related proteins in various gastric pathologies in relation to Helicobacter pylori infection: Implications in gastric carcinogenesis. Clin Res Hepato Gastroenterol 36: 235-243, 2012

13. Yang Z, Yuan XG, Chen J and Lu NH: Is NEDD4-1 a negative regulator of phosphatase and tensin homolog in gastric carcinogenesis? World J Gastroenterol 18: 6345-6348, 2012.

14. Yang Z, Yuan XG, Chen J, Luo SW, Luo ZJ and Lu NH: Reduced expression of PTEN and increased PTEN phosphorylation at residue Ser380 in gastric cancer tissues: A novel mechanism of PTEN inactivation. Clin Res Hepatol Gastroenterol 37: 72-79, 2013.

15. Li W, Xie C, Yang Z, Chen J and Lu NH: Abnormal DNA-PKes and $\mathrm{Ku} 70 / 80$ expression may promote malignant pathological processes in gastric carcinoma. World J Gastroenterol 19: 6894-6901, 2013.

16. Xie C, Xu LY, Yang Z, Cao XM, Li W and Lu NH: Expression of gammaH2AX in various gastric pathologies and its association with Helicobacter pylori infection. Oncol Lett 7: 159-163, 2014.

17. Li J, Yen C, Liaw D, Podsypanina K, Bose S, Wang SI, Puc J, Miliaresis C, Rodgers L, McCombie R, et al: PTEN, a putative protein tyrosine phosphatase gene mutated in human brain, breast, and prostate cancer. Science 275: 1943-1947, 1997.

18. Steck PA, Pershouse MA, Jasser SA, Yung WK, Lin $\mathrm{H}$, Ligon AH, Langford LA, Baumgard ML, Hattier T, Davis T, et al: Identification of a candidate tumour suppressor gene, MMAC1, at chromosome 10q23.3 that is mutated in multiple advanced cancers. Nat Genet 15: 356-362, 1997.

19. Li DM and Sun H: TEP1, encoded by a candidate tumor suppressor locus, is a novel protein tyrosine phosphatase regulated by transforming growth factor beta. Cancer Res 57: 2124-2129, 1997.

20. Sato K, Tamura G, Tsuchiya T, Endoh Y, Sakata K, Motoyama T, Usuba O, Kimura W, Terashima M, Nishizuka S, et al: Analysis of genetic and epigenetic alterations of the PTEN gene in gastric cancer. Virchows Arch 440: 160-165, 2002.

21. Byun DS, Cho K, Ryu BK, Lee MG, Park JI, Chae KS, Kim HJ and Chi SG: Frequent monoallelic deletion of PTEN and its reciprocal associatioin with PIK3CA amplification in gastric carcinoma. Int J Cancer 104: 318-327, 2003.

22. Kang YH, Lee HS and Kim WH: Promoter methylation and silencing of PTEN in gastric carcinoma. Lab Invest 82: 285-291, 2002.

23. Chun-Zhi Z, Lei H, An-Ling Z, Yan-Chao F, Xiao Y, Guang-Xiu W, Zhi-Fan J, Pei-Yu P, Qing-Yu Z and Chun-Sheng K: MicroRNA-221 and microRNA-222 regulate gastric carcinoma cell proliferation and radioresistance by targeting PTEN. BMC Cancer 10: 367, 2010

24. Xu WT, Yang Z and Lu NH: Roles of PTEN (Phosphatase and Tensin Homolog) in gastric cancer development and progression. Asian Pac J Cancer Prev 15: 17-24, 2014.

25. Yang Z, Xie C, Liu G, Cao X, Li W, Xu W and Lu N: Helicobacter pylori phosphorylates PTEN tumor suppressor to activate PI3K/Akt signaling pathway. J Gastroenterol Hepatol 28: 101, 2013.

26. Vazquez F, Ramaswamy S, Nakamura N and Sellers WR Phosphorylation of the PTEN tail regulates protein stability and function. Mol Cell Biol 20: 5010-5018, 2000.

27. Torres J and Pulido R: The tumor suppressor PTEN is phosphorylated by the protein kinase $\mathrm{CK} 2$ at its $\mathrm{C}$ terminus. Implications for PTEN stability to proteasome-mediated degradation. J Biol Chem 276: 993-998, 2001.
28. Yang Z, Xie C, Xu W, Liu G, Cao X, Li W, Chen J, Zhu Y, Luo S, Luo Z, et al: Phosphorylation and inactivation of PTEN at residues Ser380/Thr382/383 induced by Helicobacter pylori promotes gastric epithelial cell survival through PI3K/Akt pathway. Oncotarget 6: 31916-31926, 2015.

29. Tamura M, Gu J, Matsumoto K, Aota S,Parsons R and Yamada KM: Inhibition of cell migration, spreading, and focal adhesions by tumor suppressor PTEN. Science 280: 1614-1617, 1998.

30. Tamura M, Gu J, Takino T and Yamada KM: Tumor suppressor PTEN inhibition of cell invasion, migration, and growth: Differential involvement of focal adhesion kinase and p130Cas. Cancer Res 59: 442-449, 1999.

31. Tamura M, Gu J, Danen EH, Takino T, Miyamoto S and Yamada KM: PTEN interactions with focal adhesion kinase and suppression of the extracellular matrix-dependent phosphatidylinositol 3-kinase/Akt cell survival pathway. J Biol Chem 274: 20693-20703, 1999.

32. Gu J, Tamura M, Pankov R, Danen EH, Takino T, Matsumoto K and Yamada KM: Shc and FAK differentially regulate cell motility and directionality modulated by PTEN. J Cell Biol 146: 389-403, 1999.

33. Kornberg LJ: Focal adhesion kinase and its potential involvement in tumor invasion and metastasis. Head Neck 20: 745-752, 1998.

34. McLean GW, Carragher NO, Avizienyte E, Evans J, Brunton VG and Frame MC: The role of focal-adhesion kinase in cancer-a new therapeutic opportunity. Nat Rev Cancer 5: 505-515, 2005.

35. Tabassam FH, Graham DY and Yamaoka Y: OipA plays a role in Helicobacter pylori-induced focal adhesion kinase activation and cytoskeletal re-organization. Cell Microbiol 10: 1008-1020, 2008.

36. Tegtmeyer N, Wittelsberger R, Hartig R, Wessler S Martinez-Quiles N and Backert S: Serine phosphorylation of cortactin controls focal adhesion kinase activity and cell scattering induced by Helicobacter pylori. Cell Host Microbe 9 : 520-531, 2011.

37. Wang C, Yang R, Yue D and Zhang Z: Expression of FAK and PTEN in bronchioloalveolar carcinoma and lung adenocarcinoma. Lung 187: 104-109, 2009.

38. Wang SY, Hao HL, Deng K, Li Y, Cheng ZY, Lv C, Liu ZM, Yang $J$ and Pan L: Expression levels of phosphatase and tensin homolog deleted on chromosome 10 (PTEN) and focal adhesion kinase in patients with multiple myeloma and their relationship to clinical stage and extramedullary infiltration. Leuk Lymphoma 53: 1162-1168, 2012.

39. Zhang LL, Liu J, Lei S, Zhang J, Zhou W and Yu HG: PTEN inhibits the invasion and metastasis of gastric cancer via downregulation of FAK expression. Cell Signal 26: 1011-1020, 2014.

40. Kodama M, Murakami K, Nishizono A and Fujioka T: Animal models for the study of Helicobacter-induced gastric carcinoma. J Infect Chemother 10: 316-325, 2004.

41. Yan J,Luo YH and Mao YF: Establishment of Helicobacter pylori infection model in Mongolian gerbils. World J Gastroenterol 10: 852-855, 2004.

42. Myers MP, Pass I, Batty IH, Van der Kaay J, Stolarov JP, Hemmings BA, Wigler MH, Downes CP and Tonks NK: The lipid phosphatase activity of PTEN is critical for its tumor supressor function. Proc Natl Acad Sci USA 95: 13513-13518, 1998.

43. Wroblewski LE, Noble PJ, Pagliocca A, Pritchard DM, Hart CA, Campbell F, Dodson AR, Dockray GJ and Varro A: Stimulation of MMP-7 (matrilysin) by Helicobacter pylori in human gastric epithelial cells: Role in epithelial cell migration. J Cell Sci 116: 3017-3026, 2003.

44. Al-Ghoul L, Wessler S, Hundertmark T, Kruger S, Fischer W, Wunder C, Haas R, Roessner A and Naumann M: Analysis of the type IV secretion system-dependent cell motility of Helicobacter pylori-infected epithelial cells. Biochem Biophys Res Commun 322: 860-866, 2004.

45. Schneider S, Weydig C and Wessler S: Targeting focal adhesions: Helicobacter pylori-host communication in cell migration. Cell Commun Signal 6: 2, 2008.

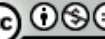

This work is licensed under a Creative Commons Attribution-NonCommercial-NoDerivatives 4.0 International (CC BY-NC-ND 4.0) License. 